\title{
Ten remarks on peptide bond formation on the ribosome
}

\author{
M.V. Rodnina ${ }^{1}$, M. Beringer and P. Bieling \\ Institute of Physical Biochemistry, University of Witten/Herdecke, 58448 Witten, Germany
}

\begin{abstract}
Peptide-bond formation is the enzymatic activity of the ribosome. The catalytic site is made up of ribosomal RNA, indicating that the ribosome is a ribozyme. This review summarizes the recent progress in understanding the mechanism of peptide bond formation. The results of biochemical and kinetic experiments, mutagenesis studies and ribosome crystallography suggest that the approx. $10^{7}$-fold rate enhancement of peptide bond formation by the ribosome is mainly due to substrate positioning within the active site, rather than to chemical catalysis.
\end{abstract}

One of the main reactions in biosynthesis of proteins is the formation of the peptide bond. Polypeptides are synthesized by the ribosome, a large particle $(2.5 \mathrm{MDa}$ in bacteria) that is made up of rRNA and proteins. The ribosome consists of two subunits, the small subunit, $30 \mathrm{~S}$, and the large subunit, $50 \mathrm{~S}$. The PTC [PT (peptidyl transferase) centre] of the ribosome is located on the $50 \mathrm{~S}$ subunit. Functionally, ribosomes are polymerases. The substrates of the reaction, peptidyl-tRNA and aminoacyl-tRNA, are bound to the $P$ and A site of the ribosome respectively. During the PT reaction, the peptide moiety is transferred from peptidyl-tRNA to aminoacyl tRNA. The chemical nature of the PT reaction is the aminolysis of an ester bond (Figure 1). The nucleophilic $\alpha$-amino group of an aminoacyl-tRNA bound to the A site of the PTC attacks the electrophilic carbonyl carbon of the ester bond linking the peptide moiety to the P-site tRNA. The resulting charged tetrahedral carbon intermediate rearranges and decomposes to yield deacylated tRNA in the $\mathrm{P}$ site and peptidyl-tRNA that is prolonged by one amino acid in the A site.

Crystal structures of the $50 \mathrm{~S}$ subunit revealed the chemical nature of the active site and indicated residues that may be involved in catalysis. The advances in ribosome genetics allowed researchers to introduce mutations in rRNA and to isolate ribosome mutants that have lethal phenotypes in vivo. Finally, biochemical and kinetic studies characterized the reaction in detail and evaluated the role of rRNA mutations and substrate substitutions. The goal of this review is to summarize these recent findings and present a unifying model of peptide-bond formation on the ribosome.

\section{Peptide-bond formation is catalysed by RNA}

The PTC is located in domain V of a $23 \mathrm{~S}$ rRNA [1]. Some PT activity was retained in $50 \mathrm{~S}$ subunits largely depleted

Key words: general acid-base catalysis, peptide-bond formation, peptidyl transferase, ribosome, ribozyme, RNA catalysis.

Abbreviations used: Pmn, puromycin; PT, peptidyl transferase; PTC, PT centre.

${ }^{1}$ To whom correspondence should be addressed (email rodnina@uni-wh.de). of protein, suggesting that the activity might reside in $23 \mathrm{~S}$ rRNA [2], although a contribution by protein could not be completely excluded [3-5]. The high-resolution crystal structures of the $50 \mathrm{~S}$ subunit from Haloarcula marismortui have revealed that the PTC is composed of RNA only, with no protein within $15 \AA\left(1 \AA=10^{-10} \mathrm{~m}\right)$ of the active site $[1,6]$ (Figure 2); a similar structure was observed in $50 \mathrm{~S}$ subunits from Deinococcus radiodurans [7]. This implies that the PT reaction is catalysed by RNA and, thus, the ribosome is a ribozyme.

\section{Residues at the active site}

The crystal structure of the $H$. marismortui $50 \mathrm{~S}$ subunit with a transition state analogue bound to the active site has revealed several ribosomal residues that could form hydrogen bonds with the reactive $\alpha$-amino group: (i) $2^{\prime}-\mathrm{OH}$ of A76 of the P site-bound peptidyl-tRNA, (ii) N3 of A2451 (Escherichia coli numbering) and (iii) $2^{\prime}-\mathrm{OH}$ of A2451 [1,8] (Figure 2). Most probably, the formation of hydrogen bonds between these groups and the $\alpha$-amino group helps to position the nucleophile; if any of these groups were to have a $\mathrm{p} K_{\mathrm{a}}$ close to neutrality, it could act as a general acid/base in the catalysis of the PT reaction. Replacement of the $2^{\prime}-\mathrm{OH}$ group of A76 in the $\mathrm{P}$ site-bound tRNA with $2^{\prime}-\mathrm{H}$ or $2^{\prime}-\mathrm{F}$ resulted in an at least $10^{6}$-fold decrease in the rate of peptide bond formation [9]. A somewhat different neighbourhood of the reactive $\alpha$-amino group was reported for a substrate analogue, i.e. Pmn (puromycin) attached to a short RNA hairpin, bound to $D$. radiodurans $50 \mathrm{~S}$ subunits [10], in contrast with results obtained from a similar substrate analogue on $H$. marismortui $50 \mathrm{~S}$ [8]. The reason for this discrepancy is not clear and the clarification awaits high-resolution ribosome structures with different substrate analogues. In addition to A2451, conserved bases U2506, U2585, C2452, A2602 and G2583 are located in the core of the PTC and may be considered as potential catalytic residues. The $50 \mathrm{~S}$ subunit structures reported do not provide any evidence for the presence of an active-site metal ion [11]. 
Figure 1 | Chemistry of peptide-bond formation

The $3^{\prime}$-ends of P-site peptidyl-tRNA (left) and A-site aminoacyl-tRNA (right) are depicted. Step 1, deprotonation of the amino group; step 2, nucleophilic attack of the $\mathrm{NH}_{2}$ group on the ester carbonyl group and formation of the zwitterionic tetrahedral intermediate, $\mathrm{T}^{ \pm}$[17]; step 3, deprotonation and formation of the negatively charged tetrahedral intermediate, $\mathrm{T}^{-}$; step 4, product formation by breakdown of the tetrahedral intermediate and protonation of the leaving oxygen. Products are P-site deacylated tRNA (left) and A-site peptidyl-tRNA (right). R1 and R2, amino acid side chains.

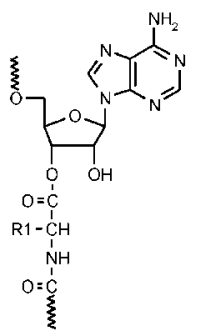<smiles>[R]C(N)C(=O)OC1C(O)C(n2cnc3c(N)ncnc32)C(O)C1CCC</smiles>

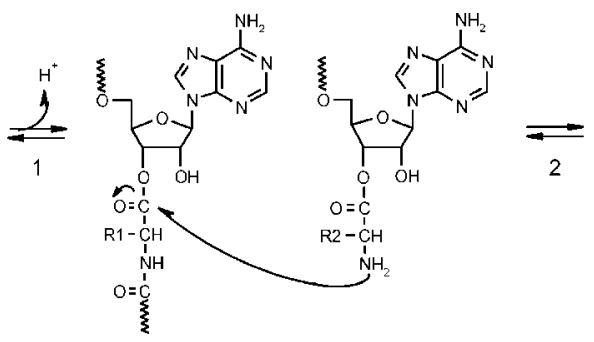

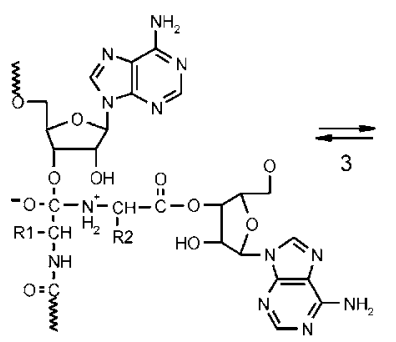

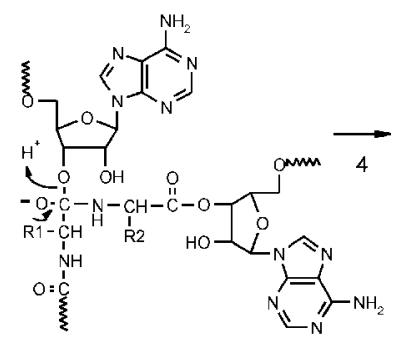<smiles>COCC1OC(O)C(O)C1n1cnc2c(N)ncnc21</smiles>

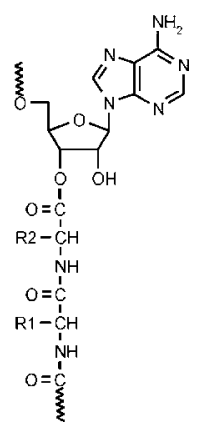

Figure 2 | The structural basis of peptide bond formation

(A) Crystal structure of the $50 \mathrm{~S}$ subunit from $\mathrm{H}$. marismortui in complex with a transition state analogue (blue) [26]. The active centre is composed of rRNA (red). Ribosomal proteins (purple) are found no closer than $18 \AA$ away from the PTC. rRNA residues at the active site are indicated (green). (B) Close-up of the active site showing the analogues of the P-site (CCA-Phe-caproic acid-biotin, Protein Data Bank accession no. 1M90; [8]) and A-site (Pmn-5'-monophosphate, PDB accession no. 1A82; [8]) substrates and the inner shell of conserved active-site nucleotides (green).
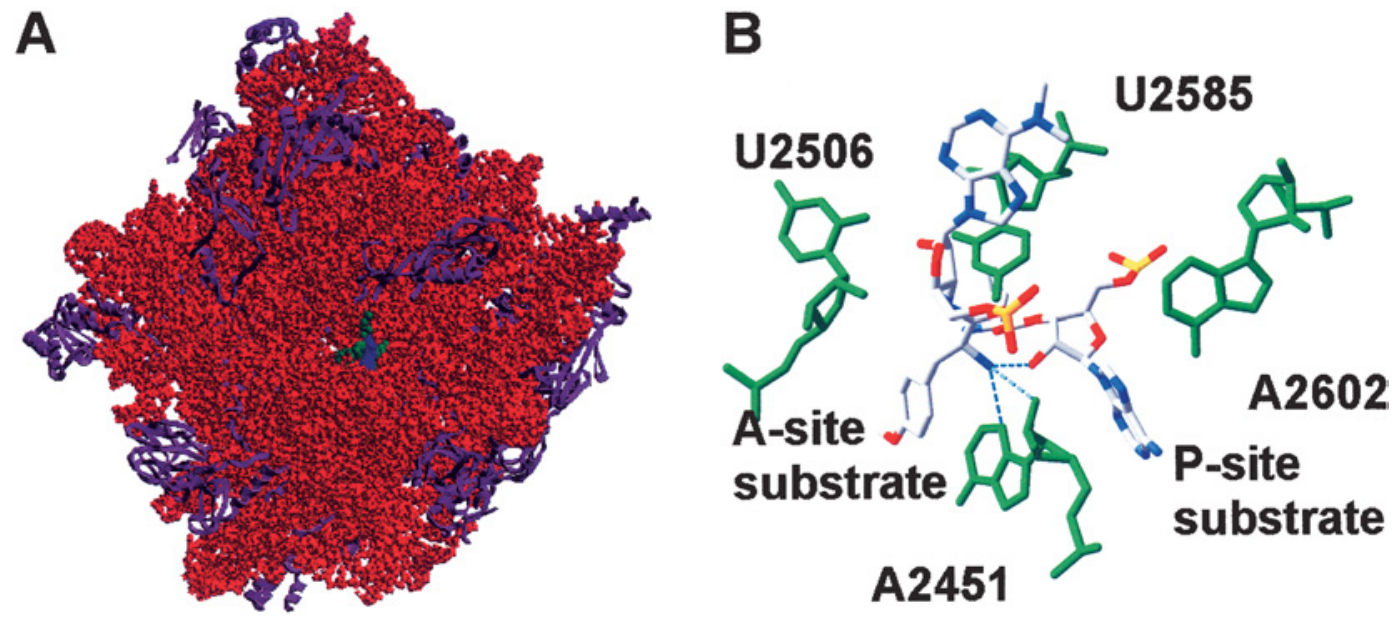

\section{Proteins are probably not involved in catalysis}

Several proteins, L2, L3 and L4, are located within a 20-30 ̊ distance from the cataly tic centre [1]. Evolutionary arguments favoured L2 as a candidate for an active site component of the PTC, since it is one of the most conserved proteins of the $50 \mathrm{~S}$ subunit [12]. Mutation of the highly conserved $\mathrm{His}^{229}$ in E. coli L2 to Glu led to $50 \mathrm{~S}$ particles devoid of 
Table 1 Activation parameters at $25^{\circ} \mathrm{C}$ for the second-order uncatalysed ester aminolysis $\left(k_{\text {non }}\right)$ and ribosome-catalysed peptide bond formation with Pmn $\left(k_{\mathrm{cat}} / K_{\mathrm{M}}\right)$ [19].

\begin{tabular}{|c|c|c|c|c|c|}
\hline \multirow[b]{2}{*}{ Amine } & \multirow[b]{2}{*}{ Ester } & \multirow{2}{*}{$\begin{array}{l}\text { Rate } \\
\left(M^{-1} \cdot s^{-1}\right)\end{array}$} & \multicolumn{3}{|c|}{$\begin{array}{l}\text { Activation parameters } \\
\text { (kcal/mol) }\end{array}$} \\
\hline & & & $\Delta G^{\neq}$ & $\Delta H^{\neq}$ & $I \Delta S^{\neq}$ \\
\hline Tris & $\begin{array}{l}\text { fGly-ethylene } \\
\text { Glycol ester }\end{array}$ & $10^{-4}$ & 22.2 & 9.1 & -13.1 \\
\hline Tris & fMet-tRNA ${ }^{\text {fMet }}$ & $10^{-4}$ & 22.7 & 16.2 & -6.5 \\
\hline Pmn & fMetPhe-tRNA ${ }^{\text {Phe }}$ & $10^{3}$ & 14.0 & 16.0 & 2.0 \\
\hline
\end{tabular}

PT activity [13]. However, other replacements of $\mathrm{His}^{229}$ have rather modest effects and the mutant ribosomes were active in the synthesis of polyphenylalanine [5], suggesting that the direct involvement of $\mathrm{His}^{229}$ of L2 in the PT reaction as a general acid/base is unlikely.

L27 is located close enough to the PTC to interact with the $\mathrm{P}$ site tRNA [7] and was shown to influence the PT activity in $E$. coli $50 \mathrm{~S}$ [14]. An L27 deletion mutant of $E$. coli is viable, but grows five to six times more slowly than the wild-type and shows deficiencies in PT activity and impaired binding of tRNA to the A site [15]. H. marismortui ribosomes do not have protein L27 or any homologous counterpart, indicating that L27 cannot be a part of an evolutionarily conserved PT mechanism which is expected to employ the same residues in all organisms. These results suggest that L27 contributes to peptide bond formation by facilitating the proper placement of the tRNA at the PTC, rather than by contributing active groups for catalysis.

\section{Characteristics of the uncatalysed reaction}

Uncatalysed model reactions for peptide bond formation were studied using different model substrates. When amino acids and aminoacyl-adenylates were used as attacking nucleophile and reactive ester respectively, the rate of reaction was very low, about $10^{-5} \mathrm{M}^{-1} \cdot \mathrm{s}^{-1}$ and varied 20 -fold depending on the identity of the amino acid, which probably reflected the considerable differences in $\mathrm{p} K_{\mathrm{a}}$ values of the attacking amino group [16]. For most nucleophiles, the ratelimiting step is the breakdown of the tetrahedral intermediate $[17,18]$. The reaction between the primary amine Tris and organic esters such as $N$-formylglycine (fGly) ethylene glycol ester $\left(10^{-4} \mathrm{M}^{-1} \cdot \mathrm{s}^{-1}\right)$ exhibited a large unfavourable activation entropy $\left(T \Delta S^{\neq}=-13.1 \mathrm{kcal} / \mathrm{mol} ; 1 \mathrm{cal}=4.184 \mathrm{~J}\right)$ and enthalpy $\left(\Delta H^{\neq}=9.1 \mathrm{kcal} / \mathrm{mol}\right)$ [19] (Table 1$)$. The reaction of Tris with a natural substrate ester of the PT reaction, fMettRNA $^{\text {fMet }}$ (where fMet stands for $N$-formylmethionine), had a more favourable entropic term and a somewhat more unfavourable activation enthalpy $\left(T \Delta S^{\neq}=-6.5 \mathrm{kcal} / \mathrm{mol}\right.$ and $\Delta H^{\neq}=16.2 \mathrm{kcal} / \mathrm{mol}$; Table 1$)$.

The PT reaction can be catalysed about 100 -fold by in vitro selected ribozymes [20]. Peptide synthesis using an in vitro selected rRNA fragment as catalyst and tRNA fragments as substrates depended on sequence complementarity between the $3^{\prime}$-CCA sequence of the aminoacyl minihelix (donor analogue) and the Pmn-bearing oligonucleotide (nucleophile analogue). However, the proximity of the reacting species was not by itself sufficient for peptide bond formation. In addition, imidazole as a catalyst was required, suggesting an importance of general acid-base catalysis for the acceleration of the PT reaction [21].

\section{Potential strategies of catalysis by the ribosome}

Charged intermediates and transition states develop during the PT reaction. First, in aqueous solution at neutral $\mathrm{pH}$, primary amines predominantly exist in the protonated ammonium form, and the $\alpha$-amino group has to be deprotonated to generate the nucleophilic $\mathrm{NH}_{2}$ group. Secondly, the putative $\mathrm{T}^{ \pm}$intermediate [17] contains a positively charged secondary amine and an oxyanion linked to the tetrahedral carbon. Thirdly, a proton is required to form the leaving group, a deacylated tRNA in the P site. One probable strategy to catalyse the reaction is to stabilize the developing charges by interactions with the ribosome due to general acid-base catalysis and electrostatic interactions. For general acidbase catalysis to occur in an aqueous environment at physiological conditions, the $\mathrm{p} K_{\mathrm{a}}$ values of the catalytic groups have to be close to neutrality to efficiently accept or donate the protons during the reaction. The $\mathrm{p} K_{\mathrm{a}}$ values of the RNA bases are, respectively, 3.5 and 4.2 for $A$ and $C$ and 9.2 for $G$ and $U$ [22]. Thus, if rRNA bases were to take part in chemical catalysis, their $\mathrm{p} K_{\mathrm{a}}$ values would have to be shifted quite significantly.

In addition to chemical catalysis, there are several other ways by which the ribosome may catalyse peptide bond formation. These include proper substrate positioning and/ or the removal of water from the active site [23-25]. Furthermore, the ribosome may stabilize the transition state by electrostatic interactions and hydrogen bonding. The ribosome may provide a preorganized electrostatic environment that decreases the activation free energy of the highly polar transition state. In particular, the negative charge on the carbonyl oxygen in the transition state could be stabilized, in analogy with the stabilization of the oxyanion in the active site of serine proteases. However, no structural or biochemical evidence for such stabilization has been found so far $[26,27]$.

\section{Contribution of general acid-base catalysis}

The rate of PT reaction was strongly $\mathrm{pH}$-dependent when using Pmn as the A-site substrate [28]. Two ionizing groups with apparent $\mathrm{p} K_{\mathrm{a}}$ values of 7.5 and 6.9 were found to contribute to the reaction. Protonation of the group with a $\mathrm{p} K_{\mathrm{a}}$ of 7.5 decreased the rate of PT reaction approx. $100-$ fold. The second $\mathrm{p} K_{\mathrm{a}}$ of 6.9 was due to the $\mathrm{NH}_{2}$ group of $\mathrm{Pmn}$, and protonation of this group eliminated the reaction almost completely. The assignment was confirmed by experiments with a Pmn derivative, Pmn-OH, in which the nucleophilic 
amino group was replaced with a hydroxy group. As expected, the rate of reaction was lower, due to lower nucleophilicity of the $\mathrm{OH}$ group compared with $\mathrm{NH}_{2}$. An ionizing group with a $\mathrm{p} K_{\mathrm{a}}$ of 7.5 was found and this group must be ribosomal, because Pmn-OH does not have a $\mathrm{p} K_{\mathrm{a}}$ close to 7.5 .

There are two important conclusions from these results. One conclusion is that in the single-protonated state, i.e. when the ribosomal group with $\mathrm{p} K_{\mathrm{a}} 7.5$ is protonated, while that with $\mathrm{p} K_{\mathrm{a}} 6.9$ is deprotonated, the reaction is still $10^{5}$-fold faster compared with the uncatalysed reaction. This part of overall catalysis may be attributed to substrate positioning, proximity effects and electrostatic stabilization of the transition state. The second conclusion is that the $\mathrm{pH}$-sensitive part of the reaction, which is due to protonation of a ribosomal group with $\mathrm{p} K_{\mathrm{a}} 7.5$, contributes another factor of 100 to the overall rate. This could be due to general acidbase catalysis. Alternatively, protonation could induce a conformation of the active site, in which the approach to the transition state is impaired for structural reasons. In such a case, mainly positional and related effects would be responsible for overall catalysis.

No pH-dependence of PT rate was observed when the natural substrate of the A site, aminoacyl tRNA, was used, or when a derivative of aminoacyl tRNA with the $\mathrm{NH}_{2}$ group replaced by an $\mathrm{OH}$ group was used (M. Beringer, P. Bieling, S. Adio and M.V. Rodnina, unpublished work). These results suggest a somewhat different PT mechanism for Pmn and aminoacyl-tRNA as the A-site substrate, and argue against an involvement of ionizing groups in the catalytic mechanism.

\section{Role of active-site residues A2451 or $2^{\prime}$-OH of A76 as general acid-base catalysts?}

To identify the groups involved in catalysis, a number of rRNA bases at the active site were mutated and the effects of replacements were examined in vivo and in vitro. $\mathrm{N} 3$ of A2451 is located within hydrogen-bonding distance from the reactive $\alpha$-amino group. Nissen et al. [29] proposed a model in which A2451 functions as a general base that abstracts a proton from the nucleophilic amino group during the formation of the tetrahedral intermediate. A general-base function of A2451 requires that the $\mathrm{p} K_{\mathrm{a}}$ of $\mathrm{N} 3$ is shifted from a very low unperturbed value towards neutrality, and it was proposed that groups in the vicinity of A2451, most importantly $\mathrm{G} 2447$, form a charge relay system that brings about the $\mathrm{p} K_{\mathrm{a}}$ shift. In its central function in forming hydrogen bonds to A2451 and to the phosphate of A2450, G2447 cannot be replaced by adenine or other bases. Therefore if the postulated general-base function of A2451 were valid, a G2447 to A mutation would be expected to eliminate acid-base catalysis and the presumed catalytic effect of the ionizing group with $\mathrm{p} K_{\mathrm{a}} 7.5$.

Mutation of A2451 to three other bases in E. coli conferred a lethal phenotype [30-32]. Nevertheless, a significant fraction of mutant ribosomes accumulated in the cells before reaching a lethal level, allowing the preparation of a mixed population of ribosomes [28,31,32] and their further affinity purification [33]. Kinetic analysis of the A2451U mutant demonstrated that peptidyl transfer to Pmn was slowed down by a factor of $150[28,33]$ and that the $\mathrm{pH}$ dependence due to the ribosomal ionizing group was eliminated [28], consistent with a direct role of A2451 in catalysis. However, mutation of G2447 had no observable effects on cell growth and was non-essential for peptide bond formation [31,32,34]. This result does not support the proposed charge relay mechanism involving G2447 and the role of A2451 as a general base in the catalysis of peptide-bond formation.

Further mutagenesis in the so-called 'inner shell' of the PTC (positions U2506, U2585 and A2602) showed that substitutions at these positions led to phenotypic effects that ranged from dominant lethality to slow growth [33]. All but one of the nine mutations exhibited a significant decrease (309400-fold compared with wild-type ribosomes) in the rate of peptide bond formation with Pmn as the A-site and fMetPhetRNA ${ }^{\text {Phe }}$ as the P-site substrate. Unexpectedly, U2506 seemed to be the most critical residue (4700-9400-fold decrease). These results indicated that the effect of A2451 mutations on peptide-bond formation was by no means exceptional and therefore argued against a role of this base as a general acidbase catalyst.

In striking contrast to the effects in the reaction with Pmn, no deficiency in the apparent rate constant for peptidyl transfer was found for any of the mutations at positions A2451, U2506, U2585 and A2602 when aminoacyl-tRNA was used as an A-site substrate [33], suggesting that none of the rRNA bases at the PTC acts in chemical catalysis of peptide bond formation. Consequently, the effects of these mutations on cell viability were attributed to an impaired peptide release induced by release factors during the termination step of protein synthesis [33].

Replacement of the 2'-OH group of A76 of P-site peptidyltRNA with $2^{\prime}-\mathrm{H}$ or $2^{\prime}-\mathrm{F}$ strongly inhibited the PT reaction, suggesting substrate-assisted catalysis [9]. The $2^{\prime}-\mathrm{OH}$ could act as a general acid donating a proton for the leaving group, the 3'-O of A76, or as a general base to deprotonate the nucleophile, the $\alpha$-amino group [9]. However, the magnitude of the rate effect (at least $10^{6}$-fold) is inconsistent with the small contribution of $\mathrm{pH}$-dependent reactions to catalysis of peptide bond formation with Pmn (a factor of 100-150) or aminoacyl-tRNA (no $\mathrm{pH}$ dependence). Thus it seems probable that $2^{\prime}-\mathrm{OH}$ of A76 is crucial for orienting the nucleophile, stabilization of the transition state or for inducing a favourable catalytic conformation of the PTC (see below).

\section{$\mathrm{pH}-$-dependent changes in ribosome structure}

The structure of PTC has long been known to be sensitive to monovalent cation concentrations and $\mathrm{pH}$. The accessibility of A2451 to chemical modification changed in response to alterations in $\mathrm{pH}$ depending on ionic conditions, temperature and the organism from which the ribosomes were obtained [30,35-37], suggesting a pH-dependent conformational change in the PTC. Interestingly, the apparent $\mathrm{p} K_{\mathrm{a}}$ of the 
rearrangement was close to 7.5 [30]. However, A2451 itself is unlikely to ionize with a $\mathrm{p} K_{\mathrm{a}}$ close to neutrality $[28,34]$. A number of residues other than A2451 also showed $\mathrm{pH}$ dependent rearrangements. Two wobble pairs, A2453-C2499 and A2450-C2063, have been proposed as potential candidates to convey pH-dependent flexibility to the PTC [28]. Each of them is presumed to possess a near-neutral $\mathrm{p} K_{\mathrm{a}}$ and both lie in proximity to A2451. The identity of the A2453C2499 bp, but not the A2450-C2063 bp, was found to be critical for the $\mathrm{pH}$-dependent structural rearrangement of A2451 [38,39]. Although the A2453-C2499 bp may be important for maintaining the structure of the active site in the E. coli PTC, its lack of conservation makes this base-pair, and consequently its near-neutral $\mathrm{p} K_{\mathrm{a}}$, unlikely to contribute to the fundamental, evolutionarily conserved mechanism of PT formation [38]. Rather, deprotonation of the base-pair may accelerate the PT reaction by stabilizing a favourable conformation of the active site, thereby contributing the observed factor of 100-150 to PT catalysis on E. coli ribosomes with Pmn as substrate [28].

\section{Contribution of substrate positioning}

Many enzymes act by lowering the activation enthalpy of the reactions they catalyse. These enthalpic sources of transition state affinity are expected for enzymes that employ general acid-base or nucleophilic catalysis to promote substrate transformation [40]. For one-substrate reactions and reactions in which the second substrate is abundant (e.g. water), the entropic contribution for the formation of the transition state is small and variable, with an average value near zero [40]. If the ribosome, like these enzymes, acted as a chemical catalyst, then the rate enhancement produced by the ribosome might be expected to arise from a decrease in the enthalpy of activation. If, on the other hand, the ribosome served mainly to position the substrates in the active site during PT, then the rate enhancement produced by the ribosome might be expected to be largely entropic in origin. In fact, the $10^{7}$-fold rate enhancement produced by the ribosome is achieved by a major lowering of the entropy of activation $\left(T \Delta S^{\neq}=2 \mathrm{kcal} / \mathrm{mol}\right.$; Table 1$)$. The enthalpy of activation is identical or slightly less favourable for the reaction on the ribosome compared with that in solution $\left(\Delta H^{\neq}=\right.$ $16.0 \mathrm{kcal} / \mathrm{mol}$ ). These results strongly support the view that the ribosome enhances the rate of peptide bond formation mainly by positioning the substrates within the active site, rather than by chemical catalysis [19]. An energetic contribution of water exclusion from the site of the reaction would also be consistent with the results.

\section{The mechanism of peptide bond formation}

The results of biochemical and kinetic experiments, mutagenesis studies and crystallography can be summarized in the following way. The ribosome is a ribozyme. It enhances the rate of peptide bond formation mainly by positioning the substrates in the active site. The possible contribution of water exclusion from the active site is unclear. In addition, the ribosome may provide a preorganized electrostatic environment that stabilizes the highly polar transition state. The contribution of general acid-base catalysis to the overall rate enhancement appears to be small. The reaction may be modulated by conformational changes at the active site, some of which are $\mathrm{pH}$-dependent.

We thank A. Kubarenko for help in preparing Figure 2. Work in our laboratory is supported by the Deutsche Forschungsgemeinschaft, the European Union, the Alfried Krupp von Bohlen und HalbachStiftung, the Bundesministerium für Bildung und Forschung and the Fonds der Chemischen Industrie.

\section{References}

1 Nissen, P., Hansen, J., Ban, N., Moore, P.B. and Steitz, T.A. (2000) Science 289, 920-930

2 Noller, H.F., Hoffarth, V. and Zimniak, L. (1992) Science 256, 1416-1419

3 Garrett, R.A. and Rodriguez-Fonseca, C. (1996) in Ribosomal RNA: Structure, Evolution, Processing, and Function in Protein Biosynthesis (Zimmermann, R.A. and Dahlberg, A.E., eds.), pp. 327-357, CRC Press, Boca Raton, FL

4 Khaitovich, P., Mankin, A.S., Green, R., Lancaster, L. and Noller, H.F. (1999) Proc. Natl. Acad. Sci. U.S.A. 96, 85-90

5 Diedrich, G., Spahn, C.M., Stelzl, U., Schafer, M.A., Wooten, T., Bochkariov, D.E., Cooperman, B.S., Traut, R.R. and Nierhaus, K.H. (2000) EMBO J. 19, 5241-5150

6 Ban, N., Nissen, P., Hansen, J., Moore, P.B. and Steitz, T.A. (2000) Science 289, 905-920

7 Harms, J., Schluenzen, F., Zarivach, R., Bashan, A., Gat, S., Agmon, I., Bartels, H., Franceschi, F. and Yonath, A. (2001) Cell 107, 679-688

8 Hansen, J.L., Schmeing, T.M., Moore, P.B. and Steitz, T.A. (2002) Proc. Natl. Acad. Sci. U.S.A. 99, 11670-11675

9 Weinger, J.S., Parnell, K.M., Dorner, S., Green, R. and Strobel, S.A. (2004) Nat. Struct. Mol. Biol. 11, 1101-1106

10 Bashan, A., Agmon, I., Zarivach, R., Schluenzen, F., Harms, J., Berisio, R., Bartels, H., Franceschi, F., Auerbach, T., Hansen, H.A. et al. (2003) Mol. Cell 11, 91-102

11 Moore, P.B. and Steitz, T.A. (2003) RNA 9, 155-159

12 Muller, E.C. and Wittmann-Liebold, B. (1997) Cell. Mol. Life Sci. 53, 34-50

13 Cooperman, B.S., Wooten, T., Romero, D.P. and Traut, R. (1995) Biochem. Cell Biol. 73, 1087-1094

14 Bischof, O., Urlaub, H., Kruft, V. and Wittmann-Liebold, B. (1995) J. Biol. Chem. 270, 23060-23064

15 Wower, I.K., Wower, J. and Zimmermann, R.A. (1998) J. Biol. Chem. 273 19847-19852

16 Weber, A.L. and Orgel, L.E. (1979) J. Mol. Evol. 13, 185-192

17 Satterthwait, A.C. and Jencks, W.P. (1974) J. Am. Chem. Soc. 96, 7018-7031

18 Jencks, W.P. and Gilchrist, M. (1968) J. Am. Chem. Soc. 90, 2622-2637

19 Sievers, A., Beringer, M., Rodnina, M.V. and Wolfenden, R. (2004) Proc. Natl. Acad. Sci. U.S.A. 101, 7897-7901

20 Zhang, B. and Cech, T.R. (1997) Nature (London) 390, 96-100

21 Tamura, K. and Schimmel, P. (2001) Proc. Natl. Acad. Sci. U.S.A. 98 1393-1397

22 Bevilacqua, P.C., Brown, T.S., Nakano, S. and Yajima, R. (2004) Biopolymers 73, 90-109

23 Krayevsky, A.A. and Kukhanova, M.K. (1979) Prog. Nucleic Acid Res Mol. Biol. 23, 1-51

24 Nierhaus, K.H. and Wittmann, H.G. (1980) Naturwissenschaften 67 234-250

25 Nierhaus, K.H., Schulze, H. and Cooperman, B.S. (1980) Biochem. Int. 1, 185-192

26 Schmeing, T.M., Seila, A.C., Hansen, J.L., Freeborn, B., Soukup, J.K., Scaringe, S.A., Strobel, S.A., Moore, P.B. and Steitz, T.A. (2002) Nat. Struct. Biol. 9, 225-230

27 Parnell, K.M., Seila, A.C. and Strobel, S.A. (2002) Proc. Natl. Acad. Sci. U.S.A. 99, 11658-11663 
28 Katunin, V.I., Muth, G.W., Strobel, S.A., Wintermeyer, W. and Rodnina, M.V. (2002) Mol. Cell 10, 339-346

29 Nissen, P., Hansen, J., Ban, N., Moore, P.B. and Steitz, T.A. (2000) Science 289, 920-930

30 Muth, G.W., Ortoleva-Donnelly, L. and Strobel, S.A. (2000) Science 289, 947-950

31 Polacek, N., Gaynor, M., Yassin, A. and Mankin, A.S. (2001) Nature (London) 411, 498-501

32 Thompson, J., Kim, D.F., O'Connor, M., Lieberman, K.R., Bayfield, M.A., Gregory, S.T., Green, R., Noller, H.F. and Dahlberg, A.E. (2001) Proc. Natl. Acad. Sci. U.S.A. 98, 9002-9007

33 Youngman, E.M., Brunelle, J.L., Kochaniak, A.B. and Green, R. (2004) Cell 117, 589-599

34 Beringer, M., Adio, S., Wintermeyer, W. and Rodnina, M.V. (2003) RNA 9 , 919-922
35 Muth, G.W., Chen, L., Kosek, A.B. and Strobel, S.A. (2001) RNA 7 , 1403-1415

36 Xiong, L., Polacek, N., Sander, P., Bottger, E.C. and Mankin, A. (2001) RNA 7, 1365-1369

37 Bayfield, M.A., Dahlberg, A.E., Schulmeister, U., Dorner, S. and Barta, A (2001) Рroc. Natl. Acad. Sci. U.S.A. 98, 10096-10101

38 Bayfield, M.A., Thompson, J. and Dahlberg, A.E. (2004) Nucleic Acids Res. 32, 5512-5518

39 Hesslein, A.E., Katunin, V.I., Beringer, M., Kosek, A.B., Rodnina, M.V. and Strobel, S.A. (2004) Nucleic Acids Res. 32, 3760-3770

40 Wolfenden, R., Snider, M., Ridgway, C. and Miller, B. (1999) J. Am. Chem. Soc. 121, 7419-7420

Received 28 January 2005 